\title{
Iniciativas empresariales de segunda oportunidad: Situación, expectativas y contexto financiero a nivel internacional
}

\author{
Second chance business initiatives: \\ Situation, expectations and international financial context
}

Rubén Lado-Sestayo

Universidad de la Coruña, España

Milagros Vivel-Búa

Universidad de Santiago de Compostela, España

Autor para correspondencia: ruben.lado.sestayo@udc.es

Fecha de recepción: 13 de julio 2017 - Fecha de aceptación: 30 de noviembre de 2017

Resumen: Este trabajo analiza las diferencias en las empresas creadas por reemprendedores y el resto de emprendedores en cuatro dimensiones. Así, se analiza si la situación de la empresa, las expectativas de crecimiento, la situación financiera del emprendedor y la financiación de las inversiones presentan diferencias estadísticamente significativas. El trabajo se ha realizado en una muestra amplia del sector turístico utilizando los microdatos del Global Entrepreneurship Monitor (GEM) para el período 2007-2013. Asimismo, las iniciativas empresariales se han clasificado en tres grupos atendiendo al nivel de desarrollo de la economía, de acuerdo con la clasificación utilizada por el Foro Económico Mundial (FEM). Los resultados señalan diferencias importantes en las empresas creadas por emprendedores y re-emprendedores, y el hecho de que dichas diferencias varíen en función del nivel de desarrollo económico.

Palabras clave: segunda oportunidad; contexto financiero; emprendimiento

\begin{abstract}
This paper analyzes the differences in companies created by re-entrepreneurs and the rest of entrepreneurs in four dimensions. Thus, it is analyzed if the situation of the company, the expectations of growth, the financial situation of the entrepreneur and the financing of the investments present statistically significant differences. The work has been done in a large sample of the tourism sector using microdata from the Global Entrepreneurship Monitor (GEM) for the period 2007-2013. Likewise, business initiatives have been classified into three groups according to the level of development of the economy, according to the classification used by the World Economic Forum (WEF). The results indicate important differences in the companies created by entrepreneurs and re-entrepreneurs, and the fact that these differences vary depending on the level of economic development.
\end{abstract}

Key words: second chance; financial context; entrepreneurship 


\section{Introducción}

La crisis económica ha supuesto un importante cambio estructural en diversas economías, afectando notablemente al nivel de empleo y de bienestar social y a las iniciativas empresariales (Soininen, Puumalainen, Sjögrén \& Syrjä, 2012). Asimismo, su impacto ha sido mayor en aquellos sectores cíclicos, como lo es el sector turístico, donde ha generado importantes cierres empresariales y afectado a multitud de iniciativas empresariales, entre ellas microempresas (Lado-Sestayo et al.; Vivel-Búa \& Otero-González, 2017). La recuperación de la actividad económica requiere recuperar inversiones y de nuevo poner en marcha iniciativas empresariales. Por ello, se han puesto en marcha programas para el fomento de la actividad emprendedora en multitud de países, con el doble objetivo de reducir el desempleo e incrementar la innovación y la competitividad de la economía (Minniti, 2008; Leitão \& Baptista, 2009). Para el correcto diseño de estas medidas es necesario conocer el impacto que la crisis económica ha ocasionado sobre los potenciales emprendedores y sobre las iniciativas empresariales. En particular, es importante señalar que el estigma que ocasiona el cierre de una empresa puede impactar significativamente en las características de una nueva apertura (Simmons, Wiklund \& Levie, 2014). Así, el objetivo de este trabajo es analizar las particularidades de las iniciativas creadas por emprendedores que han cerrado una iniciativa recientemente en el sector turístico considerando el nivel de desarrollo de su economía. El estudio de estas diferencias constituye una novedad a la literatura, puesto que los únicos trabajos centrados en el impacto de este estigma se han realizado sin centrarse en un sector y en economías particulares.

La capacidad del sector turístico de generar riqueza y bienestar ha sido demostrada en numerosos estudios, por lo que constituye uno de los principales mecanismos para el fomento de medidas de crecimiento económico. No obstante, es importante señalar que el actual contexto de crisis que atraviesan ciertas economías afecta especialmente a la obtención de financiación. En consecuencia, el estudio de las diferencias en las características de las iniciativas creadas por el colectivo de reemprendedores debe tener en cuenta no solo características empresariales, tales como el nivel de competencia o la estructura organizativa, sino que especialmente debe considerar las expectativas y el contexto financiero.

Este trabajo para alcanzar su objetivo utiliza los microdatos disponibles en el Global Entrepreneurship Monitor (GEM) para el período 2007-2013. Así, se han identificado reemprendedores del sector turístico en economías orientadas a factores, a la eficiencia y a la innovación, de acuerdo con clasificado establecida por el Foro Económico Mundial (FEM). Este criterio analiza una serie de pilares de las economías, entre los que se encuentran las instituciones, las infraestructuras, la estabilidad macroeconómica, la salud y educación primaria, la educación superior y capacitación, la eficiencia en el mercado de bienes, la eficiencia en el mercado laboral, la sofisticación del mercado financiero, la preparación tecnológica, el tamaño del mercado, la sofisticación empresarial y la innovación. De este modo, el contexto de cada reemprendedor es tenido en cuenta a la hora de analizar sus diferencias respecto al conjunto de emprendedores, lo que resulta útil para considerar diferencias a la hora de diseñar medidas de fomento económico orientadas a incrementar el reemprendimiento.

Este trabajo se estructura del siguiente modo: Tras la sección de introducción se presenta la revisión de la literatura, donde se destacan los pocos estudios que analizaron el 
reemprendimiento. Posteriormente se expone el análisis empírico que se divide en tres secciones. Así, en primer lugar, se presenta la base de datos utilizada, el proceso de construcción de la muestra y la metodología utilizada. A continuación, en segundo lugar, se realiza un análisis descriptivo tanto del reemprendimiento como de las variables analizadas. Finalmente, en tercer lugar, se presentan las principales diferencias obtenidos entre emprendedores y reemprendedores en las distintas economías analizadas. El trabajo termina con la presentación de las principales conclusiones extraídas, sus limitaciones y líneas de trabajo futuro.

\section{Revisión de la Literatura}

Existe una amplia literatura sobre el estudio de los factores que determinan la actividad emprendedora y sus características, especialmente de carácter reciente (Busenitz, Plummer, Klotz, Shahzad \& Rhoads, 2014). El aumento de la importancia de la actividad emprendedora como motor económico puede explicar este creciente interés desde la academia por su comprensión. Sin embargo, pocos son los estudios centrados en el sector turístico, y la mayor parte de los mismos han analizado los factores que determinan la decisión de emprender de los individuos (Ateljevic, 2009; Ramos-Rodríguez, Medina-Garrido, \& Ruiz-Navarro, 2012). Asimismo, recientemente algunos estudios analizan no los individuos sino los factores de la empresa creada y la incidencia del entorno sobre las mismas (Lado-Sestayo et al.; Vivel-Búa \& Otero-González, 2017). Esta literatura ha contribuido a trasladar el marco teórico sobre los factores que inciden en el emprendimiento al sector, confirmando su validez en el mismo.

El estudio del reemprendimiento es muy reciente, y la literatura es muy escasa. Asimismo, es importante destacar que los reemprendedores presentan particularidades que deben ser tenidas en cuenta (Hsu, Wiklund \& Cotton, 2017). Así, su conocimiento de la actividad emprendedora es mayor, lo cual puede ser una ventaja frente al resto de emprendedores y consecuentemente afectar a las características de la empresa creada (Minniti \& Bygrave, 2001; Yamakawa, Peng \& Deeds, 2015). En este sentido, los recursos y capacidades de los que dispone el reemprendedor contribuyen a que se encuentre en una mejor situación frente al resto de emprendedores (Silver, 2015). Sin embargo, el cierre de una iniciativa empresarial puede ocasionar un estigma, el cual se ve a su vez afectado por el entorno socioeconómico y cultural del país, por lo que debe ser considerado. En consecuencia, estudios focalizados en una única economía podrían no ser directamente extrapolables (Pilková, Holienka \& Munk, 2013; Munk, Pilková \& Holienka, 2014). De este modo, es importante considerar en el estudio el estigma (Simmons, Wiklund \& Levie, 2014), y particularmente centrar la atención en las diferencias entre distintos contextos económicos y socio-culturales.

En relación a los condicionantes del entorno, estudios previos señalan como uno de los principales factores que afectan al resultado de las iniciativas empresariales en el sector turístico el nivel de competencia (Lado-Sestayo, Otero-González, Vivel-Búa \& Martorell-Cunill, 2016). Asimismo, el estigma creado puede afectar a cuestiones organizativas de la empresa, a las expectivas y a los recursos de los que disponen los reemprendedores, tales como las redes (Simmons, Wiklund, \& Levie, 2014). Finalmente, debe considerarse que estas limitaciones y recursos se han producido en el último año en un contexto de crisis que ha limitado financiación. 
Los estudios sobre el reemprendimiento son muy escasos y ninguno centrado en el sector turístico. Así, Wagner (2002) analiza las respuestas de 1.000 personas mayores de 14 años en Alemania. Sus resultados apuntan a que tanto los factores individuales como regional afectan significativamente a la decisión de reemprender. Asimismo, los autores reconocen que el estigma del fracaso empresarial en Alemania podría ser más elevado que en otros países, de modo que los resultados deben ser tomados con cautela. Landier (2005) analiza datos de Francia del 1990 al 2000 en emprendedores que pasan a ser empleados en el siguiente período. Mientras que Evans and Leighton (1989) and Hamilton (2000) encuentran que los emprendedores que abandonan y pasan a ser empleados ganan más sueldo en Estados Unidos, Landier (2005), encuentra para Francia el resultado contrario. Así, el autor señala que diferencias en el estigma del fracaso entre ambos países podría explicar estas diferencias.

Stam, Audretsch \& Meijaard (2008) analizan 240 respuestas de exemprendedores en un panel de los años 1994, 1998, 1999, 2000 y 2004 para analizar su intención de emprender de nuevo. En su estudio encuentran que los factores determinantes de la decisión de emprender son los mismos en emprendedores y reemprendedores, si bien estos modifican su importancia. Asimismo, los autores encuentran que los reemprendedores presentan más experiencia y más recursos que los emprendedores que no han fracaso previamente. Sin embargo, Nielsen \& Sarasvathy (2016) analizan una muestra en Dinamarca de 1980 al 2007. Los autores encuentran que la decisión de reemprender por parte de emprendedores fracasados puede provocar un mercado de limones (Akerlof, 1970). En este contexto, se dificulta la obtención de financiación por parte de los emprendedores exitosos.

Algunos estudios afirman que el entorno cultural y económico, por ejemplo, derivado de una economía socialista, puede tener un impacto en la decisión de reemprender (Pilková, Holienka \& Munk, 2013; Munk, Pilková \& Holienka, 2014). A nuestro juicio, estos son los únicos trabajos en esta área y consecuentemente existe en la literatura una falta de trabajos focalizados en el análisis del reemprendimiento turístico. Por ello, diversos autores destacan la necesidad de más estudios, los cuales deben considerar especialmente las condiciones sectores y del entorno (Yamakawa, Peng, \& Deeds, 2015; Hsu, Wiklund, \& Cotton, 2017); Lado-Sestayo, Vivel-Búa \& Otero-González, 2017).

Este trabajo contribuye a la literatura al ser la primera evidencia empírica que analiza las diferencias en las iniciativas creadas por emprendedores y reemprendedores. En particular, se presta especial atención a las diferencias de la situación de la empresa, sus expectativas, la situación financiera del emprendedor y el contexto de financiación de la empresa. Los resultados son útiles para diseñar medidas específicas orientadas a este colectivo, en un sector con un impacto elevado en la economía como lo es el sector turístico.

\section{Análisis Empírico}

\section{Presentación de la Base de Datos y la Metodología}

Este trabajo utiliza los microdatos del proyecto GEM para el período 2007-2013. Nos es posible incorporar períodos más recientes debido a que los microdatos posterior a 2013 no son públicos. El proyecto GEM es un consorcio en el que se incluyen investigadores de todo el 
mundo que tiene como objetivo analizar el fenómeno emprendedor en las distintas economías. Para ello, el proyecto realiza un número muy elevado de encuestas en todo el mundo.

Entre las preguntas realizadas se pide a los emprendedores que manifiesten su actividad y esta se codifica. Gracias a ello, ha sido posible identificar los siguientes códigos ISIC rev3: 5510, 5520, 6304, 9232 y 9233 y códigos ISIC rev4 de 5510, 5520, 5590, 5610, 5621, 5629, 5630, $7911,7912,7990$ y 9102, que se corresponden con los sectores que componen el sector turístico al incluir empresas de alojamiento, restauración, organizadoras de viajes y servicios culturales de acceso a centros de interés. De estas actividades, se han identificado aquellos emprendedores que forman parte de lo que se conoce como emprendimiento incipiente, de modo que se han obtenido un total de 7.465 emprendedores incipientes en el sector turístico en todo el mundo. De ellos, un total de 764 son emprendedores que manifestaron haber cerrado una iniciativa empresarial en los últimos 12 meses.

Las iniciativas empresariales y sus emprendedores han sido clasificados en tres grupos de acuerdo con la categoría de la economía en la que desarrollaban su actividad. Para ello, se ha tenido en cuenta la clasificación del Foro Económico Mundial entre países orientados a factores, países orientados a la eficiencia y países orientados a la innovación. Una vez clasificados, se ha realizado un análisis dentro de cada categoría, con el objetivo de disminuir así las diferencias derivadas de los distintos niveles de desarrollo económico en el que se realiza la actividad.

Para evaluar las diferencias entre las características de la iniciativa empresarial creada entre emprendedores y reemprendedores se han utilizado tres test estadísticos, atendiendo a la naturaleza de la variable. Así, para variables continuas se ha utilizado un test t de diferencia de medidas, mientras que para las variables categóricas se ha utilizado un test Chi2 de diferencia de proporciones. Finalmente, para las variables numéricas que representan unidades monetarias se ha realizado el test $\mathrm{K}$ no paramétrico de diferencia de medianas.

\section{Análisis Descriptivo}

La variable dependiente utilizada "reemprendedor" es una variable dicotómica que toma el valor 1 si el emprendedor manifiesta que la iniciativa empresarial se pone en marcha tras haber cerrado una iniciativa en los últimos 12 meses y toma el valor 0 si el emprendedor manifiesta que no ha cerrado una iniciativa en los últimos 12 meses. Un análisis de esta variable para los tres grupos de economías identificados (factores, eficiencia, innovación) señala que la principal motivación para cerrar la anterior iniciativa fue que esta no era rentable. A continuación, se observan ciertas diferencias, ya que mientras las razones personales es la segunda causa en las economías orientadas a los factores y la eficiencia, en las economías orientadas a la innovación lo son otros motivos. En tercer lugar, para los países orientados a la eficiencia y en cuarto lugar para los países orientados a los factores y a la innovación se encuentran los problemas financieros. Las diferencias en las motivaciones son estadísticamente significativas de acuerdo con el test Chi2 de diferencia de proporciones.

En conjunto, se observa una presencia mucho mayor de reemprendedores entre los emprendedores en las economías orientadas a factores, donde representan un $15,83 \%$, mientras que en los países orientados a la eficiencia representan un 10,83\% y en los países orientados a la innovación representan un $6,81 \%$. 


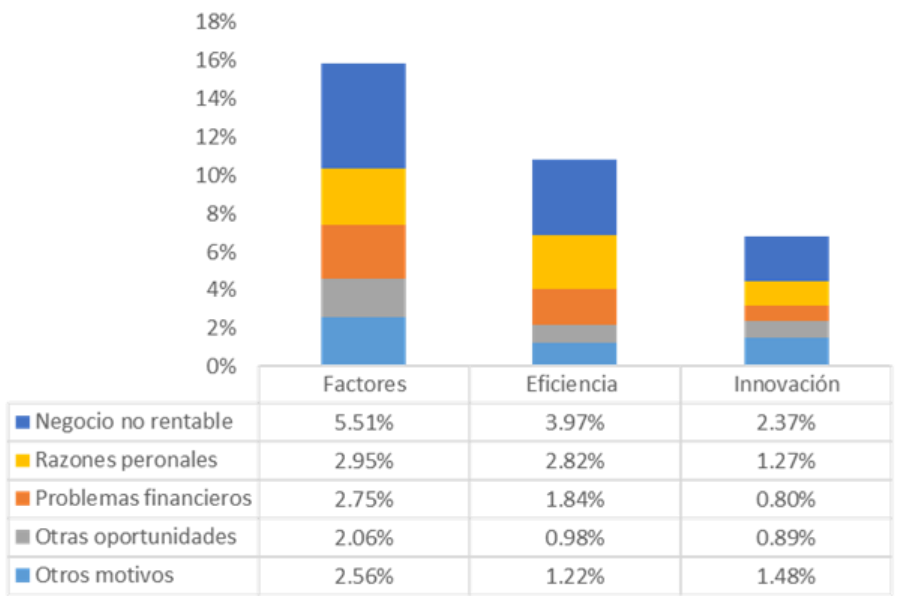

Figura 1. Motivaciones del abandono de la anterior iniciativa empresarial

Las características empresariales se han clasificado en cuatro bloques, dentro de las cuales se han identificado un conjunto de variables:

1. Situación de la empresa

a. Novedad: Es una variable que toma tres valores en función de los potenciales clientes que consideran el producto o servicio novedoso.
i. Todos
ii. Alguno
iii. Ninguno

b. Competencia: Es una variable que toma tres valores en función del número de empresas que ofrecen el mismo producto.
i. Muchas
ii. Alguna
iii. Ninguna

c. Gestión: Es una variable dicotómica que toma el valor 1 si la gestión de la empresa es compartida.

2. Expectativas

a. Tamaño: Indica el tamaño que el emprendedor espera que tenga la empresa en 5 años. Toma 4 posibles valores:

i. 0 empleados

ii. Entre 1 y 5 empleados

iii. Entre 6 y 19 empleados

iv. Más de 19 empleados

b. Crecimiento: Es una variable dicotómica que toma el valor 1 si el emprendedor espera que el número de empleados aumente más de 10 y más de un $50 \%$ en los próximos 5 años.

3. Situación financiera del emprendedor

a. Necesidad: Es una variable dicotómica que toma el valor 1 si el emprendedor indica que la motivación para crear la empresa es la necesidad.

b. Ingresos: Es una variable que toma tres valores en función de los ingresos del emprendedor dentro del país: 
i. Tercio con menores ingresos

ii. Tercio con ingresos medios

iii. Tercio con mayores ingresos

c. Financiador: Es una variable dicotómica que toma el valor 1 si el emprendedor financia otras iniciativas empresariales

4. Contexto de financiación de la empresa

a. Inversión: Es una variable que indica la inversión necesaria inicial de la empresa.

b. Aportación: Es una variable que indica la inversión necesaria inicial que el emprendedor ha tenido que desembolsar para poner en marcha la empresa

c. Peso_capital: Es una variable que indica el porcentaje que representa la aportación del emprendedor sobre la inversión total necesaria para poner en marcha la empresa.

d. Externos: Es una variable que indica el número de propietarios de la empresa.

\section{Resultados}

A continuación, se expone el análisis de diferencias entre emprendedores y reemprendedores. En relación con la situación de la empresa, se observa que las diferencias son significativas respecto a la menor presencia de gestión compartida en las economías orientadas a la eficiencia por parte de los reemprendedores frente al resto de emprendedores. Para las otras variables las diferencias obtenidas no son estadísticamente significativas entre emprendedores y reemprendedores (Tabla 1). En lo relativo a las expectivas, las empresas de los reemprendedores esperan en mayor medida mantenerse con un reducido tamaño en los próximos 5 años en las economías orientadas a los factores. Por el contrario, esperan en mayor medida presentar un mayor tamaño en las economías orientadas a la innovación. Asimismo, en las economías orientadas a factores hay una menor expectativa de alto crecimiento en los reemprendedores, un $38 \%$ inferior frente al resto de emprendedores. Por el contrario, en las economías orientadas a la eficiencia las expectativas de alto crecimiento son un $23 \%$ superiores en los reemprendedores y un $60 \%$ superiores en los reemprendedores de países orientados a la innovación. Ello podría deberse a que el capital humano y social adquirido en la etapa anterior podría presentar un distinto efecto en función de la situación de la economía y existir diferencias en las oportunidades de crecimiento en función del nivel de desarrollo de la economía (Tabla 2).

Tabla 1. Diferencias en la situación de la empresa entre emprendedores y reemprendedores. Análisis por clasificación de la economía de acuerdo con el FEM

\begin{tabular}{|c|c|c|c|c|c|c|c|c|c|c|}
\hline \multirow{2}{*}{\multicolumn{2}{|c|}{ Situación de la empresa }} & \multicolumn{3}{|c|}{ Factores } & \multicolumn{3}{|c|}{ Eficiencia } & \multicolumn{3}{|c|}{ Innovación } \\
\hline & & & & & & & & & & \\
\hline \multirow{3}{*}{ Novedad } & Ninguno & $61.29 \%$ & $6522 \%$ & 0.93 & $4860 \%$ & $4751 \%$ & 084 & $6083 \%$ & $5714 \%$ & 098 \\
\hline & Alguno & $23.39 \%$ & $20.50 \%$ & $(0.63)$ & $28.24 \%$ & $27.38 \%$ & $(0.65)$ & $22.97 \%$ & $24.22 \%$ & $(0.61)$ \\
\hline & Todos & $15.32 \%$ & $14.29 \%$ & & $23.16 \%$ & $25.11 \%$ & & $16.21 \%$ & $18.63 \%$ & \\
\hline \multirow[t]{3}{*}{ Competencia } & Ninguno & $7.37 \%$ & $8.07 \%$ & & $10.28 \%$ & $10.63 \%$ & 1.05 & $10.99 \%$ & $12.42 \%$ & 4.60 \\
\hline & Alguno & $31.93 \%$ & $26.71 \%$ & & $36.19 \%$ & $33.71 \%$ & $(0.59)$ & $33.05 \%$ & $24.84 \%$ & $(0.100)$ \\
\hline & Todos & $60.70 \%$ & $65.22 \%$ & & $53.53 \%$ & $55.66 \%$ & & $55.97 \%$ & $62.73 \%$ & \\
\hline Gestión & $\%$ & $61.97 \%$ & $72.67 \%$ & $\begin{array}{c}1.73 \\
(0.42)\end{array}$ & $57.74 \%$ & $52.26 \%$ & $\begin{array}{c}4.83 \\
(0.03)\end{array}$ & 57.2 & 60.87 & $\begin{array}{c}0.81 \\
(0.37)\end{array}$ \\
\hline
\end{tabular}


Tabla 2. Diferencias en las expectativas entre emprendedores y reemprendedores. Análisis por clasificación de la economía de acuerdo con el FEM

\begin{tabular}{|c|c|c|c|c|c|c|c|c|c|c|}
\hline \multicolumn{2}{|c|}{ Expectativas } & \multicolumn{3}{|c|}{ Factores } & \multicolumn{3}{|c|}{ Eficiencia } & \multicolumn{3}{|c|}{ Innovación } \\
\hline & & No & Sí & $\begin{array}{l}\text { T (p- } \\
\text { val) }\end{array}$ & No & Sí & $\begin{array}{l}\text { T (p- } \\
\text { val) }\end{array}$ & No & Sí & $\begin{array}{l}\text { T (p- } \\
\text { val) }\end{array}$ \\
\hline Tamaño & $\begin{array}{c}0 \\
1-5 \\
6- \\
19\end{array}$ & $\begin{array}{l}10.63 \% \\
52.30 \% \\
30.03 \%\end{array}$ & $\begin{array}{l}15.27 \% \\
59.54 \% \\
19.85 \%\end{array}$ & $\begin{array}{c}7.66 \\
(0.05)\end{array}$ & $\begin{array}{c}9.02 \% \\
52.72 \% \\
26.89 \%\end{array}$ & $\begin{array}{c}8.63 \% \\
49.87 \% \\
26.68 \%\end{array}$ & $\begin{array}{c}3.93 \\
(0.27)\end{array}$ & $\begin{array}{l}13.75 \% \\
56.85 \% \\
21.34 \%\end{array}$ & $\begin{array}{l}12.86 \% \\
48.57 \% \\
22.86 \%\end{array}$ & $\begin{array}{l}10.66 \\
(0.01)\end{array}$ \\
\hline Crecimiento & $\begin{array}{c}>19 \\
\%\end{array}$ & $\begin{array}{c}7.04 \% \\
27.71 \%\end{array}$ & $\begin{array}{c}5.34 \% \\
17.11 \%\end{array}$ & $\begin{array}{c}3.75 \\
(0.05)\end{array}$ & $\begin{array}{l}11.37 \% \\
40.32 \%\end{array}$ & $\begin{array}{l}14.82 \% \\
49.25 \%\end{array}$ & $\begin{array}{c}5.90 \\
(0.01)\end{array}$ & $\begin{array}{c}8.07 \% \\
26.93\end{array}$ & $\begin{array}{c}15.71 \% \\
43.06\end{array}$ & $\begin{array}{c}8.60 \\
(0.00)\end{array}$ \\
\hline
\end{tabular}

Respecto a las diferencias en la financiación, se observa que en las economías orientadas a factores hay más reemprendimiento por necesidad, que alcanza casi la mitad de los reemprendedores. En cuanto a su nivel de ingresos, se observa en las economías orientadas a eficiencia que se encuentran en los menores inferiores de renta, mientras que en las economías orientadas a la innovación ocurre justo lo contrario. Finalmente, independientemente del estado de desarrollo de la economía se observa que en los reemprendedores exista una presencia mucho mayor de business angel, es decir, de personas que invierten en otras iniciativas empresariales. El conocimiento del mercado y de la actividad emprendedora adquirida en la etapa anterior, así como la búsqueda de la diversificación podrían explicar esta mayor presencia de los reemprendedores en esta actividad (Tabla 3). Finalmente, la inversión de la nueva iniciativa es menor en los reemprendedores de las economías orientadas a factores frente al resto de emprendedores, y aportan menor cantidad con sus fondos propios, lo que puede indicar que buscan una menor exposición al riesgo de fracasar. Por el contrario, en las economías orientadas a la innovación los fondos aportados por los reemprendedores son mayores que los aportados por los emprendedores. La proporción de la inversión que es asumida por el reemprendedor es menor en las economías orientadas a la eficiencia, lo que podría indicar que en estas economías la experiencia como emprendedor contribuye a la obtención de financiación de terceros.

Finalmente, en todas las economías hay una mayor concentración de la propiedad de la empresa en los reemprendedores, lo que puede indicar que estos deciden compartir en menor medida los ingresos esperados o que disponen de los conocimientos necesarios $\mathrm{y}$, consecuentemente, no necesitan socios para la puesta en marcha de la empresa (Tabla 4).

Tabla 3. Diferencias en la situación financiera entre emprendedores y reemprendedores. Análisis por clasificación de la economía de acuerdo con el FEM

\begin{tabular}{|c|c|c|c|c|c|c|c|c|c|c|}
\hline \multirow{2}{*}{\multicolumn{2}{|c|}{$\begin{array}{c}\text { Situación } \\
\text { financiera }\end{array}$}} & \multicolumn{3}{|c|}{ Factores } & \multicolumn{3}{|c|}{ Eficiencia } & \multicolumn{3}{|c|}{ Innovación } \\
\hline & & No & Sí & $\begin{array}{l}\text { T (p- } \\
\text { val) }\end{array}$ & No & Sí & $\mathrm{T}$ (p-val) & No & Sí & $\begin{array}{l}\text { T (p- } \\
\text { val) }\end{array}$ \\
\hline Necesidad & $\%$ & $\begin{array}{c}32.71 \\
\%\end{array}$ & $\begin{array}{c}39.75 \\
\%\end{array}$ & $\begin{array}{c}3.00 \\
(0.08 \\
\quad) \\
\end{array}$ & $\begin{array}{c}30.04 \\
\%\end{array}$ & 33.26 & $1.93(0.17)$ & 19.34 & 22.36 & $\begin{array}{c}0.87 \\
(0.35 \\
\quad)\end{array}$ \\
\hline
\end{tabular}




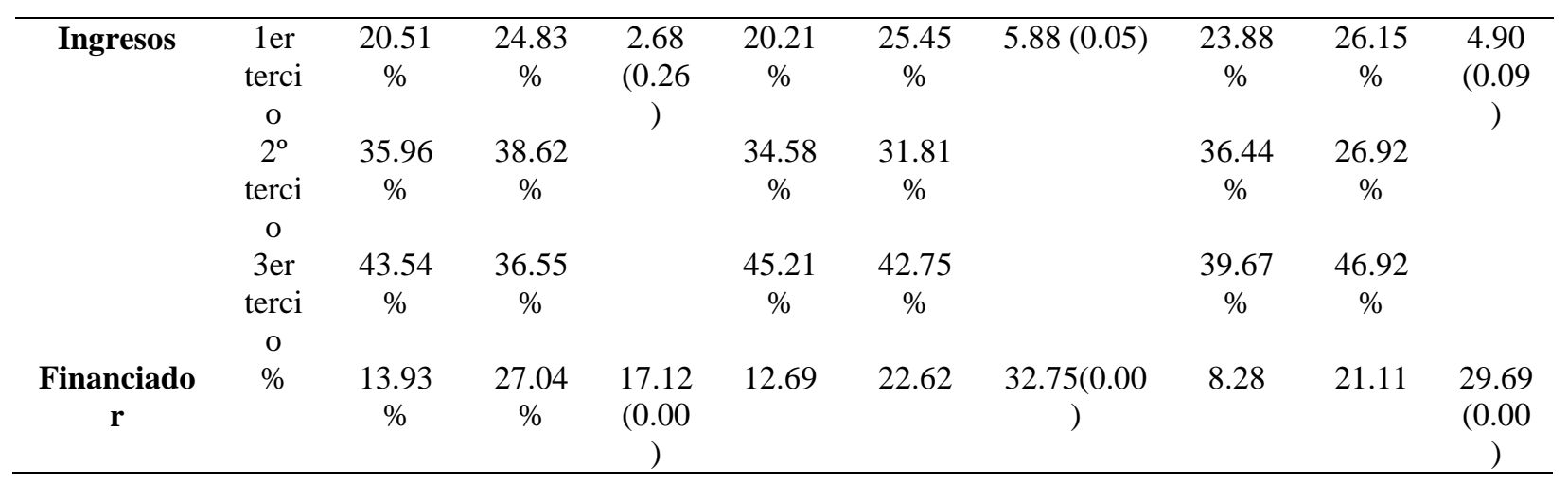

Tabla 4. Diferencias en la financiación de la empresa entre emprendedores y reemprendedores. Análisis por clasificación de la economía de acuerdo con el FEM

\begin{tabular}{|c|c|c|c|c|c|c|c|c|c|c|}
\hline \multirow{2}{*}{\multicolumn{2}{|c|}{ Financiación }} & \multicolumn{3}{|c|}{ Factores } & \multicolumn{3}{|c|}{ Eficiencia } & \multicolumn{3}{|c|}{ Innovación } \\
\hline & & No & Sí & $\mathrm{T}$ (p-val) & No & Sí & $\mathrm{T}$ (p-val) & No & Sí & $\mathrm{T}$ (p-val) \\
\hline Inversión & Median & 2.000 & 300 & $\begin{array}{l}18.93 \\
(0.00)\end{array}$ & 50 & 50 & $\begin{array}{l}23.16 \\
(0.63)\end{array}$ & 50 & 80 & $\begin{array}{c}1.97 \\
(0.16)\end{array}$ \\
\hline Aportación & Median & 650 & 350 & $\begin{array}{c}4.06 \\
(0.04)\end{array}$ & 50 & 37.5 & $\begin{array}{c}0.04 \\
(0.84)\end{array}$ & 24 & 50 & $\begin{array}{c}3.34 \\
(0.07)\end{array}$ \\
\hline Peso_capital & Media & $47.46 \%$ & $54.42 \%$ & $\begin{array}{c}-0.78 \\
(0.45)\end{array}$ & 49.55 & 44.15 & $\begin{array}{c}2.05 \\
(0.04)\end{array}$ & 46.30 & 47.75 & $\begin{array}{c}-0.30 \\
(0.76)\end{array}$ \\
\hline Externos & $\begin{array}{c}\text { Sd } \\
\text { Media } \\
\text { Sd }\end{array}$ & $\begin{array}{c}8.11 \% \\
42.08 \%\end{array}$ & $\begin{array}{c}8.50 \% \\
33.33 \%\end{array}$ & $\begin{array}{c}2.81 \\
(0.09)\end{array}$ & $\begin{array}{c}5.83 \\
46.48\end{array}$ & $\begin{array}{c}4.97 \\
40.75\end{array}$ & $\begin{array}{c}4.04 \\
(0.05)\end{array}$ & $\begin{array}{c}6.74 \\
54.65\end{array}$ & $\begin{array}{c}7.82 \\
45.13\end{array}$ & $\begin{array}{c}3.81 \\
(0.05)\end{array}$ \\
\hline
\end{tabular}

En síntesis, y tratando de sintetizar un perfil por estado de desarrollo de la economía, se observa que los emprendedores frente a los emprendedores presentan las siguientes diferencias (Tabla 5).

Tabla 5. Síntesis de las diferencias encontradas entre reemprendedores y emprendedores

\begin{tabular}{|c|c|c|}
\hline Economías orientadas a factores & Economías orientadas a eficiencia & $\begin{array}{l}\text { Economías orientadas a la } \\
\text { innovación }\end{array}$ \\
\hline -Menores expectativas de & -Menor gestión compartida & \multirow{4}{*}{$\begin{array}{l}\text {-Mayores expectativas de } \\
\text { crecimiento } \\
\text {-Mayor nivel de ingresos }\end{array}$} \\
\hline crecimiento & -Mayores expectativas de & \\
\hline -Mayor motivación por necesidad & crecimiento & \\
\hline -Mayor presencia de Business & -Menor nivel de ingresos & \\
\hline Angels & -Mavor presencia de Business & \multirow{2}{*}{$\begin{array}{c}\text {-Mayor presencia de Business } \\
\text { Angels }\end{array}$} \\
\hline -Menor inversión & Angels & \\
\hline -Menor capital aportado & -Mayor financiación de terceros & \multirow{2}{*}{$\begin{array}{l}\text {-Mayor capital aportado } \\
\text {-Menor número de socios }\end{array}$} \\
\hline & & \\
\hline
\end{tabular}

\section{Conclusiones}

En este trabajo se han analizado las diferencias entre los emprendedores y reemprendedores en cuatro dimensiones, que se corresponden con situación de la empresa, expectativas, situación financiera del emprendedor y financiación de las inversiones. El trabajo 
se ha realizado para el período 2007-2013 en una muestra amplia del sector turístico. Asimismo, el análisis ha considerado tres estados distintos de desarrollo de la economía, de acuerdo con el Foro Económico Mundial (FEM) entre los que se encuentran economías orientadas a factores, economías orientadas a la eficiencia y economías orientadas a la innovación. Los resultados obtenidos señalan importantes diferencias entre emprendedores y reemprendedores en diversas variables, y dichas diferencias dependen del estado de desarrollo de la economía. Estos resultados son útiles para conocer las causas del reemprendimiento turístico y para el diseño de medidas de fomento de esta actividad. Asimismo, existe una falta importante de estudios en esta área de acuerdo con la literatura, de modo que este trabajo contribuye a este gap.

Los resultados obtenidos señalan que, en las economías orientadas a factores, los reemprendedores presentan menores expectivas de crecimiento, lo que podría explicarse por la mayor presencia de iniciativas creadas por necesidad. A este respecto, es importante analizar el tipo de empresa creada a la hora de fomentar dichas actividades, ya que muchas podrían estar actuando como un sustituto de la falta de alternativas laborales. Asimismo, estas iniciativas presentan una menor inversión y un menor capital aportado, lo que podría ser una señal de una menor confianza del emprendedor en el éxito de la nueva empresa o bien podrían indicar que la anterior iniciativa empresarial supone una merma de recursos. Así, la mejora de las leyes de bancarrota, junto con apoyos financieros específicos que fomenten la participación con fondos propios podrían ser medidas para este tipo de economías.

En las economías orientadas a la eficiencia se observa menor propensión a compartir la gestión, por lo que la experiencia previa puede aportar conocimientos en esta área. Asimismo, se presentan mayores expectativas de crecimiento, lo que podría deberse a las oportunidades que presentan economías emergentes, tales como Brasil, Chile o México. Sin embargo, el reemprendimiento se genera en mayor medida en los segmentos de renta baja, por lo que la merma de recursos como consecuencia del fracaso anterior podría explicar este hecho. Asimismo, debe notarse que la mayor presencia de financiación de terceros podría indicar facilidades de crédito en dichas economías, ya que atraen una suma importante de inversión directa extranjera, y en este contexto, los reemprendedores podrían presentar mejores recursos y capacidades por su experiencia previa.

En las economías orientadas a la innovación, los reemprendedores presentan mayores expectativas de crecimiento y mayor nivel de ingresos. Sin embargo, aportan menos capital sobre el total de la inversión requerida. La existencia de oportunidades de inversión en sectores con altas posibilidades y baja inversión tales como aquellos relacionados con el uso de las TIC, la economía colaborativa o la explotación del big data podrían explicar este efecto. En este contexto, las ventajas de conocimiento y experiencia de los reemprendedores podrían ser una ayuda para anticiparse a estos cambios recientes en el mercado. Así, el establecimiento de programas de mentoring podría ser una alternativa para el fomento de la actividad emprendedora. En esta línea, independientemente del nivel de desarrollo de la economía se observa que los reemprendedores son más propensos a participar en iniciativas empresariales de terceros. Además, presentan recursos y capacidades por su anterior experiencia, por lo que medidas que permitan trasladar y compartir dicho conocimiento podrían ser de interés para incrementar la actividad emprendedora y consecuentemente la generación de actividad económica. 
Entre las principales limitaciones encontradas está el hecho de que no ha sido posible obtener información más completa sobre la iniciativa creada, ni tampoco sobre la iniciativa anterior. Así, la relación entre la actividad cerrada anteriormente y la nueva actividad podría ser objeto de estudio en trabajos anteriores. De este modo, se podría evaluar en qué medida el conocimiento y experiencia adquiridos se relaciona con la nueva actividad. Otra de las limitaciones encontradas hace referencia al tipo de información. A este respecto, se trata de encuestas personales, de modo que la falta de respuesta en algunas variables, como por ejemplo en lo relativo a la inversión realizada, podría estar sesgada en los valores más elevados. De este modo, es necesario validar estos resultados utilizando datos reales de la empresa que no sean facilitados por el emprendedor, sino que provengan de fuentes públicas y objetivas, tales como los registros contables. Finalmente, el estudio no ha sido posible realizarlo exclusivamente para un país debido a que se reduciría notablemente el tamaño muestral. Consecuentemente, es importante para controlar adecuadamente el estigma social del fracaso realizar estudios dentro del mismo país y misma cultura, lo cual podría ser objeto de estudio de trabajos posteriores.

\section{Bibliografía}

Akerlof, G. A. (1970). The market for" lemons": Quality uncertainty and the market mechanism. The quarterly journal of economics, 488-500. http://www.jstor.org/stable/1879431

Ateljevic, J. (2009). Tourism entrepreneurship and regional development: example from New Zealand. International Journal of Entrepreneurial Behavior \& Research, 15(3), 282-308. http://www.emeraldinsight.com/doi/abs/10.1108/13552550910957355

Busenitz, L. W., Plummer, L. A., Klotz, A. C., Shahzad, A., \& Rhoads, K. (2014). Entrepreneurship research (1985-2009) and the emergence of opportunities. Entrepreneurship Theory and Practice, 38(5), 981-1000. http://onlinelibrary.wiley.com/doi/10.1111/etap.12120/full

Evans, D. S., \& Leighton, L. S. (1989). Some empirical aspects of entrepreneurship. The American Economic Review, 79(3), 519-535. http://www.jstor.org/stable/1806861

Hamilton, B. H. (2000). Does entrepreneurship pay? An empirical analysis of the returns to selfemployment. Journal of Political economy, 108(3), 604-631. http://www.journals.uchicago.edu/doi/abs/10.1086/262131

Hsu, D. K., Wiklund, J., \& Cotton, R. D. (2017). Success, Failure, and Entrepreneurial Reentry: An Experimental Assessment of the Veracity of Self- Efficacy and Prospect Theory. Entrepreneurship Theory and Practice, 41(1), 19-47. http://onlinelibrary.wiley.com/doi/10.1111/etap.12166/full

Lado-Sestayo, R., Vivel-Búa, M., \& Otero-González, L. (2017). Drivers and barriers to entry for new hotel $\begin{array}{llll}\text { start-ups. } & \text { Tourism } & \text { Management } & \text { Perspectives, }\end{array}$ http://www.sciencedirect.com/science/article/pii/S2211973617300430

Lado-Sestayo, R., Otero-González, L., Vivel-Búa, M., \& Martorell-Cunill, O. (2016). Impact of location on profitability in the Spanish hotel sector. Tourism Management, 52, 405-415. http://www.sciencedirect.com/science/article/pii/S0261517715001648

Landier, A. (2005), Entrepreneurship and the Stigma of Failure. Available at SSRN: http://dx.doi.org/10.2139/ssrn.850446 
Leitão, J., \& Baptista, R. (Eds.). (2009). Public policies for fostering entrepreneurship: A European perspective (Vol. 22). Springer Science \& Business Media. https://link.springer.com/book/10.1007/978-1-4419-0249-8

Minniti, M. (2008). The role of government policy on entrepreneurial activity: productive, unproductive, or destructive?. Entrepreneurship Theory and Practice, 32(5), 779-790. http://onlinelibrary.wiley.com/doi/10.1111/j.1540-6520.2008.00255.x/full

Minniti, M., \& Bygrave, W. (2001). A dynamic model of entrepreneurial learning. Entrepreneurship: Theory and practice, $25(3), 5-5$. https://elibrary.ru/item.asp?id=6369407

Munk, M., Pilková, A. \& Holienka, M. (2014): Business Restart in Visegrad Countries. Entrepreneurial Business and Economics Review 21, 37-48. https://www.ceeol.com/search/article-detail?id=67770

Nielsen, K., \& Sarasvathy, S. D. (2016). A market for lemons in serial entrepreneurship? Exploring type I and type II errors in the restart decision. Academy of Management Discoveries, 2(3), 247-271. https://amd.aom.org/content/2/3/247.short

Pilková, A., Holienka, M., \& Munk, M. (2013). Key business restart drivers in Slovakia. Acta Universitatis Agriculturae et Silviculturae Mendelianae Brunensis, 61(7), 2617-2622. http://dx.doi.org/10.11118/actaun201361072617

Ramos-Rodríguez, A. R., Medina-Garrido, J. A., \& Ruiz-Navarro, J. (2012). Determinants of hotels and restaurants entrepreneurship: A study using GEM data. International Journal of Hospitality Management, $31(2)$, 579-587. http://www.sciencedirect.com/science/article/pii/S0278431911001381

Silver, L. S. (2015). A social-cognitive model of entrepreneurship failure: Beyond attributional style. Archives of Business Research, 3(4). http://www.sseuk.org/index.php/ABR/article/view/1306

Simmons, S. A., Wiklund, J., \& Levie, J. (2014). Stigma and business failure: implications for entrepreneurs' career choices. Small Business Economics, 42(3), 485-505. https://link.springer.com/article/10.1007/s11187-013-9519-3

Soininen, J., Puumalainen, K., Sjögrén, H., \& Syrjä, P. (2012). The impact of global economic crisis on SMEs: does entrepreneurial orientation matter?. Management Research Review, 35(10), 927-944. http://www.emeraldinsight.com/doi/abs/10.1108/01409171211272660

Stam, E., Audretsch, D., \& Meijaard, J. (2008). Renascent entrepreneurship. Journal of Evolutionary Economics, 18(3-4), 493-507.Evans, D. S., \& Leighton, L. S. (1989). Some empirical aspects of entrepreneurship. The American Economic Review, 79(3), 519-535. https://link.springer.com/article/10.1007/s00191-008-0095-7

Wagner, J. (2002): Taking a Second Chance: Entrepreneurial Restarters in Germany, IZA Discussion paper series, No. 417. Available at: http://hdl.handle.net/10419/20029

Yamakawa, Y., Peng, M. W., \& Deeds, D. L. (2015). Rising from the ashes: Cognitive determinants of venture growth after entrepreneurial failure. Entrepreneurship Theory and Practice, 39(2), 209-236. http://onlinelibrary.wiley.com/doi/10.1111/etap.12047/full 